\title{
AUTOBIOGRAFISMO E DIALOGO FILOSOFICO: IL CASO STORIOGRAFICO DE LA FESTA DI NATALE DI FRIEDRICH SCHLEIERMACHER
}

\author{
Francesca D'Alberto ${ }^{1}$
}

\begin{abstract}
RIASSUNTO: Die Weihnachtsfeier. Ein Gespräch di Friedrich Schleiermacher è uno degli esempi più interessanti di come, nel romanticismo, forma letteraria, contesto vitale e autobiografico, tesi filosofiche costituissero un complesso unitario e spesso di difficile interpretazione. Nel caso del dialogo schleiermacheriano sul Natale è possibile distinguere due livelli di lettura storiografica: uno, rivolto soprattutto al contesto, consente di identificare i riferimenti biografici e l'ambiente reale a cui allude l'autore; un secondo, strettamente legato al primo, ma non necessariamente riducibile ad esso, ha invece un valore, per così dire, "autointerpretativo". Alla luce di questo secondo livello di lettura, questa breve opera di Schleiermacher mostra di essere ben più di uno scritto occasionale per il Natale del 1806. Esso nasconde e rivela al contempo, nelle trame di un gioco di rimandi e allusioni biografiche, la volontà di rottura con il circolo romantico, a cui pure il dialogo è dedicato, e segna l'inizio per l'autore di una riflessione nuova e autonoma.
\end{abstract}

PAROLE CHIAVE: Friedrich Schleiermacher. Religione. Arte. Musica. Romanticismo.

\section{INTRODUZIONE}

Nel romanticismo, che intreccia riflessione filosofica, profonde e spesso contraddittorie forme di religiosità, sperimentazione letteraria, l'impianto sistematico - sia dal punto di vista della costruzione del pensiero sia dal punto di vista del tipo di scrittura utilizzata per dare forma al sistema si va affermando solo lentamente, fino a imporsi in modo definitivo con i sistemi idealistici. Tuttavia, come ben testimonia, ad esempio, la produzione schellinghiana, prima di "cristallizzarsi" in forme sistematiche, la scrittura filosofica aveva sviluppato, in epoca romantica e preromantica, numerose possibilità alternative e si era intimamente legata alla riflessione sull'arte. Non a caso, il recupero della filosofia e della letteratura greca prestava ai romantici numerosi modelli di scrittura filosofica, che andavano ad arricchire il panorama della saggistica, della pubblicistica e della "pamphlettistica" di matrice illuministica. È sufficiente ricordare a questo proposito le numerose iniziative

\footnotetext{
${ }^{1}$ Dipartimento FISPPA. Università degli Studi di Padova. francesca.dalberto@unipd.it
} 
letterarie "sperimentali", per così dire, di Friedrich Schlegel per richiamare alla mente l'atmosfera di forte innovazione letteraria tra fine Settecento e inizio Ottocento e la tenace volontà di reinterpretare il rapporto tra filosofia, arte e creatività individuale.

Nel clima spumeggiante del romanticismo, che si cimentava in tutti gli ambiti della produzione e della riflessione artistica, trova spazio un'opera assai particolare ed enigmatica, che riassume in sé gli aspetti tipici di questa sperimentazione di fine Settecento e al contempo presenta una serie di difficoltà interpretative che ne fanno ancor oggi un "oggetto" piuttosto controverso. Si tratta dello scritto Die Weihnachtsfeier. Ein Gespräch di Friedrich Schleiermacher, il teologo riformato che visse pienamente la stagione preromantica e fu fedele amico del suo maggior rappresentante, appunto il già citato Friedrich Schlegel. L'opera in questione, pubblicata in prima edizione nel 1806 e in seconda edizione nel 1826, nonostante il grande successo editoriale, fu oggetto di numerose recensioni negative da parte dei romantici, di cui pure Schleiermacher era compagno e amico, e di una assai positiva recensione invece da parte di Schelling. Già questo accenno alla ricezione de La festa di Natale da parte dei contemporanei dell'autore mette sulle tracce delle difficoltà che hanno accompagnato i tentativi di decifrare il carattere dell'opera e di trovarne la giusta collocazione nell'orizzonte della discussione filosofica dei primi dell'Ottocento.

In questo contributo si cercherà, intrecciando elementi testuali e contestuali, di offrire uno dei (numerosi) possibili accessi al suo significato.

\section{ANONIMATO, SORPRESA E GESELLIGKEIT}

Come già era accaduto per le Reden über die Religion e per i Monologen, anche in occasione di Die Weihnachtsfeier Schleiermacher decise di pubblicare in forma anonima la sua opera ${ }^{2}$. E, come per i Monologen, egli concepì il suo scritto come un regalo agli amici per una precisa circostanza: i Monologhi, scritti per il Capodanno del 1799, erano da intendersi come un dono per l'anno nuovo, con il quale iniziava anche un nuovo secolo, occasione che ben si prestava quindi a riflessioni etiche sui mali dell'epoca. La festa di Natale è

${ }^{2}$ SCHLEIERMACHER, Friedrich. Über di Religion. Reden an die Gebildeten unter ihren Verächtern, Berlin 1799 (tr. it., Sulla religione. Discorsi a quegli intellettuali che la disprezzano, a cura di Salvatore Spera. Brescia: Queriniana, 2005²; Id., Monologen. Ein Neujahrsgabe, Berlin: Spener, 1800 (tr.it., Monologhi, a cura di Ferruccio Andolfi. Reggio Emilia: Diabasis, 2011). 
intesa come un dono per una ben determinata cerchia di amici in occasione della festività religiosa.

Tanto la forma anonima quindi, quanto l'opera intesa come dono per qualcuno erano due elementi caratteristici della scrittura schleiermacheriana nell'epoca in cui l'autore era partecipe dei destini del movimento romantico.

Nello specifico, La festa di Natale fu composta da Scheiermacher durante il periodo trascorso a Halle dall'ottobre 1804 fino al 1807. A Halle Schleiermacher era attivo presso la facoltà luterana come professore di filosofia e teologia, e si dedicò soprattutto ad approfondire la sua già ampia riflessione etica, tenendo nuovi corsi sulla dottrina delle virtù. Accanto a questi è importante ricordare i corsi sulla Lettera a Timoteo, nei quali Schleiermacher dava esempio del lavoro di critica storico-letteraria intorno ai testi biblici, mettendo al centro dell'interpretazione l'esperienza umana fonte vitale e concreta delle espressioni letterarie.

$\mathrm{Al}$ di là della attività accademica e omiletica alla quale Schleiermacher si dedicò in questo periodo - durante il quale, cosa assai importante per la nostra ricostruzione, continuò ad applicarsi alla traduzione di Platone, che è stata, non occorre ricordarlo, la traduzione classica, tuttora riferimento d'obbligo, per la ricezione di Platone in Germania - fu la conoscenza di nuove persone (in primo luogo Henrik Steffens) e la frequentazione di salotti e cerchie sociali sconosciuti (la famiglia Reichardt in particolare) a segnare profondamente anche la sua produzione filosofica e letteraria, come è tipico di tutta la sua attività di pensatore, profondamente segnata dalle relazioni di amicizia e dai legami affettivi più significativi. Filosofia e Geselligkeit, pensiero e socievolezza, vanno perciò tenuti presenti entrambi quando si prendono in considerazione gli scritti di Schleiermacher, il cui pensiero è sempre congiunto a un preciso contesto sociale e alla riflessione sull'etica e sui costumi che in tale contesto prendono forma. Non si può dunque prescindere, nella lettura del dialogo sul Natale, dalle circostanze e dall'ambito in cui esso è nato.

Se si cerca dunque l'occasione concreta che ha spinto Schleiermacher a comporre questo dialogo, si risale alla sera del 2 dicembre 1805, quando il filosofo ebbe l'opportunità di ascoltare il concerto del flautista Friedrich Ludwig Dülon, allora molto conosciuto e apprezzato. Una volta concepita l'idea del dialogo - non a caso nato dall'ascolto della musica, più che da una riflessione teologica ad hoc sul significato della natività - egli cercò di farlo pubblicare per Natale, riuscendo però a inviarlo all'editore solo la mattina del 
24 dicembre, così che esso apparve nel gennaio dell'anno successivo soltanto a Halle, Berlino e Rügen. In queste tre località, non a caso, alloggiavano gli amici di Schleiermacher. Già questo indizio rivela lo scopo primo di quest'opera: per quanto essa abbia avuto in seguito, come sopra accennato, un notevole successo editoriale e una notevole diffusione, originariamente essa venne pensata dal suo autore per un contesto ben definito di persone.

Duplice era dunque l'intento di questo dono anonimo: da un lato, Schleiermacher voleva comprendere se i destinatari avrebbero indovinato chi aveva fatto loro questo dono, e quindi chi aveva composto il dialogo; dall'altro voleva verificare se avrebbero correttamente interpretato i riferimenti concreti che venivano fatti nel dialogo stesso.

Tutti questi elementi rendono pienamente lecito interpretare lo scritto in termini squisitamente biografici: anzi, cercare i diretti riferimenti di Schleiermacher e le persone reali che si nascondono dietro i protagonisti del dialogo è necessario anche per comprenderne l'idea filosofica centrale. Tanto più che questo gioco di svelamento (che richiama, come spesso è stato messo in luce anche dai contemporanei dell'autore, il senso del cognome Schleiermacher, "facitore di veli") viene scherzosamente ripreso in tutte le prime pagine del dialogo, nelle quali la caccia ai doni di Natale si svolge in una sorta di penombra che vela e svela contemporaneamente l'oggetto cercato:

[...] l'accogliente sala era ornata a festa [...] le numerose lampade accese, appese in alto, diffondevano una luce festosa, che però stuzzicava furbescamente la curiosità. Essa, infatti, permetteva di vedere abbastanza nitidamente le cose familiari, mentre quelle sconosciute o nuove solo lentamente e con un'attenzione più insistente potevano venire individuate con precisione $[\ldots]$ in modo che solo lentamente l'impazienza, eccitata in parte per scherzo e in parte seriamente, venisse soddisfatta, e i piccoli doni colorati restassero avvolti ancora per un breve momento da un'aura che ne accresceva il fascino ${ }^{3}$.

È questo l'incipit del dialogo, che conduce il lettore nel pieno di una festa intima e familiare, ma al contempo misteriosa, nella quale l'accoglienza amichevole e il clima confidenziale della casa si intrecciano con la ricerca di qualcosa di nascosto. ${ }^{3}$ SCHLEIERMACHER, Friedrich. La festa di Natale: un dialogo. A cura di Giovanni Moretto. Brescia:
Queriniana, 1994, p. 61-62. 
Questo intreccio di anonimità, riferimenti biografici mascherati, elementi scherzosi e piacere del dono - tutti elementi comuni alla Romantik - implica il fatto di rivolgersi a un cerchia precisa di amici intimi: in tal senso uno scritto quasi esoterico come quello qui analizzato si presta ad essere compreso solo da un gruppo di "iniziati” all'amicizia .

Ma c'è ancora qualcosa in più in questa breve composizione: se i riferimenti biografici ivi contenuti consentono di ricostruire al meglio l'ambiente familiare di una casa borghese con la quale Schleiermacher aveva consuetudine, più complesso è comprendere il valore filosofico che l'autore attribuiva al dialogo. Non si tratta di uno scritto divulgativo sul Natale ad opera di un teologo ben conosciuto (e poco amato, a Halle, in verità): come si è sottolineato nelle pagine precedenti, il carattere esoterico dello scritto, avvalorato tanto dagli elementi biografici quanto dalla forma anonima, esclude che si tratti di un dialogo a uso di un ampio pubblico. Né il pubblico a cui era destinata l'opera era quello dei teologi. L'intento schleiermacheriano, come si è visto, era di parlare a pochi e di farsi comprendere da quei pochi con i quali aveva condiviso un'intimità tale da render evidente ciò che ad altri rimaneva oscuro. Non si trattava dunque per Schleiermacher né di argomentare il senso teologico del Natale né di divulgare al grande pubblico un messaggio universale. Il messaggio che passa attraverso questo dialogo è in primo luogo un messaggio personale, che riguarda la vita dell'autore, in secondo luogo un messaggio filosofico, che riguarda il suo pensiero. E come si è già sottolineato, vita e pensiero erano per Schleiermacher un'unica cosa.

Dal punto di vista filosofico non è di secondaria importanza il fatto che, in quegli anni, egli andasse traducendo i dialoghi platonici, nonché progettasse di scrivere egli stesso dei dialoghi nei quali le posizioni della filosofia contemporanea fossero "mascherate" $\mathrm{da}$ personaggi tratti dall'antichità. Questo espediente, in parte retorico (se visto alla luce di quell'esoterismo che caratterizzava il modo di scrivere dei romantici), allude anche ad una possibile commistione di forme platoniche (dialogo) e aristoteliche (discussione delle differenti posizioni ${ }^{5}$ ). Insieme agli elementi biografici dal contesto, va quindi

\footnotetext{
${ }^{4}$ Giovanni Moretto, nella sua introduzione all'opera, fa presente questi tratti esoterici dello scritto, che richiedevano quasi una "decifrazione" del testo (Cf. MORETTO, Giovanni. Etica e narrazione nella Festa di Natale di Schleiermacher. In: SCHLEIERMACHER, Friedrich. La festa di Natale: un dialogo, op. cit., p. 11-12), e rimanda agli studi di Hermann Patsch, Die esoterische Kommunikationsstruktur der "Weihnachtsfeier". Über Anspielungen und Zitate. In:. RICHARDSON, R. D. (Ed.). Schleiermacher in context. Lewiston: Edward Mellen Press,1991, p. 132-156.
}

${ }^{5}$ Come fa notare Moretto nell'introduzione, op. cit., p. 28. 
tenuta presente l'intenzione filosofica con la quale Schleiermacher affrontava i temi presentati in occasione del Natale e di cui la forma dialogica è parte essenziale.

\section{IL CONTESTO: IL LIVELLO BIOGRAFICO}

Come sopra accennato, La festa di Natale è ambientata nell'abitazione di una famiglia borghese che si riunisce insieme agli amici più stretti per la festività. Oltre agli adulti, che si alternano nella conversazione, secondo un modello che ricorda esplicitamente il Simposio platonico, sono presenti due bambini, Sofia, figlia di Ernestina e il fratellino.

Sofia è una figura chiave del dialogo, in quanto i temi fondamentali in esso affrontati - il rapporto di musica e religione, la nascita e la maternità, il ruolo della donna nella vita sacra - vengono alla luce principalmente attraverso le parole e i comportamenti della bambina. È Sofia, infatti, a mettere in scena la natività: attraverso una sorta di presepe meccanico, Sofia rappresenta non solo la nascita di Gesù, ma l'intera storia del cristianesimo, dal battesimo di Cristo passando per Lutero fino alle vicende dell'orfanotrofio (Waisenhaus) di Halle che, sottolinea l'autore, ella "[...] aveva considerato come l'ultima grande opera dell'entusiasmo religioso". ${ }^{6}$

La rappresentazione religiosa di tipo plastico, sottolinea l'autore, costituisce agli occhi di Sofia un livello ancora insoddisfacente per la sua volontà di esprimere il sentimento religioso: è nella musica, infatti, che la bambina cerca l'espressione più appropriata della religiosità, tanto che, tra $\mathrm{i}$ regali ricevuti, subito si appassiona al libro di composizioni musicali natalizie donatele dal padre. Il suo talento per la musica, ci viene detto "[...] è altrettanto grande quanto limitato": Sofia, infatti, "[...] non può eseguire con facilità se non ciò che si presenta nel grande stile ecclesiastico".

Con questa bambina dai tratti così particolari e tutt'altro che infantili, in realtà, il tema della musica, che aveva ispirato Schleiermacher a scrivere il dialogo dopo aver udito il concerto di Dülon, viene introdotto in stretto legame con la religiosità e, soprattutto, con la religiosità pietistica di Herrnhut, comunità all'interno della quale era cresciuto lo stesso autore. Alla musica vengono intimamente collegati, tramite la figura di Sofia, la gioia della

${ }^{6}$ La festa di Natale, op. cit., p. 69.

${ }^{7}$ Ibid., p. 67. 
fanciullezza e la maternità. Fanciullezza, maternità, musica e gioia semplice del canto costituiscono lo sfondo entro il quale prendono forma i successivi racconti e discorsi.

\section{LA STRUTTURA}

\subsection{RACCONTI DELLE DONNE}

Di parole, infatti, si tratta nel prosieguo dell'opera: dapprima le donne, considerate, fin dall'apertura del dialogo, come le protagoniste di questa festa, come le custodi dell'armonia familiare e dei valori della natività, narrano storie vissute relative al Natale.

Mentre Federica accompagna i racconti delle donne al pianoforte, Ernestina, la padrona di casa, propone il primo racconto, in cui richiama la figura di una donna con il figlio in braccio. Il bambino, ci descrive la narratrice, era "[...] preso da un semiconscio colloquio, fatto di amore e nostalgia, con la madre" . Per Ernestina era questa una "[...] delle figure vive quali belle immagini di Maria e del Bambino" 9 . Tale rappresentazione vivente fortemente simbolica, spiega la donna, altri non era che Cornelia, la sorella di Edoardo: il figlio, qui ricordato quando era ancora bambino, era morto poi in guerra.

A questo primo racconto di maternità e dolore, segue la narrazione di Agnese, che fa da controcanto a quella di Ernestina, alternando alla mestizia, già introdotta dal racconto precedente, la gioia del Natale, con un evidente richiamo allo stile "musicale" e all'alterarsi in esso di tonalità emotive contrastanti. Ricordando il battesimo del bimbo della sorella la sera di Natale, Agnese sottolinea che, nei doni portati al fanciullo, "[...] erano sorprendentemente mescolati lo scherzo e la serietà, come si addice ad ogni allusione al futuro" ${ }^{10}$. Il senso di questa narrazione, riassunto "speculativamente" da Leonardo come immagine di "[...] un Cristo bambinello negativo, rovesciato, nel quale l'aureola, invece che verso l'esterno, proietta la sua luce verso l'interno", sta invece nelle parole di Agnese, che così apostrofa Leonardo:

[...] tu ora puoi anche dire in modo migliore e più bello di quanto non sia capace io, anzi sei il solo in grado di dire perché io vi abbia raccontato tutto ciò proprio questa sera. Io, infatti, non so descrivere a parole quanto profondamente e intimamente abbia sentito allora che ogni gioia serena è

\footnotetext{
${ }^{8}$ Ibid., p. 103.

${ }^{9}$ Ibid.

${ }^{10}$ Ibid., p. 108.
} 
religione, che amore, letizia e devozione sono suoni di un'armonia perfetta, che ad ogni modo possono susseguirsi e scontrarsi ${ }^{11}$.

La natura musicale della religione, richiamata da Agnese, colora anche il terzo racconto, un racconto di speranza e nuova vita, proposto da Carolina. Raccontando la guarigione inaspettata del figlio di Carlotta, avvenuta a Natale, Carolina mette in luce, anche in questo frangente, il mescolarsi dei differenti toni che caratterizzano il Natale. E così la madre del figlio malato, ci narra, era piena "[...] di malinconia e insieme di dolcezza al pensiero di inviare in cielo un angelo nei giorni in cui celebriamo l'invio sulla terra dell'angelo più grande di tutti"12. Con l'esito positivo e la guarigione del figlio, il bambino "[...] resta un angelo [...] purificato dalla sofferenza [...] passato attraverso la morte per venire consacrato a una vita nuova" ${ }^{13}$.

Dopo una breve interruzione, segnata dall'arrivo di nuovi conoscenti, prende avvio la terza parte del dialogo, dopo quella introduttiva, raccolta intorno alla figura di Sofia, e dopo quella dei racconti delle donne, incentrata sostanzialmente sulla musicalità della religione e sulla levità dei toni che in essa si alternano, e che consentono di esprimere un sentimento preciso, una gioia tutta speciale, differente da quella che si prova in altre occasioni di festa. Questa terza parte è dedicata ai discorsi.

\subsection{DISCORSI DEGLI UOMINI}

Su suggerimento di Ernesto viene proposto di scegliere un argomento sul quale ognuno sia tenuto a dire qualcosa. Non dunque a narrare, ma ad argomentare. La festa di Natale viene scelta come argomento dei discorsi e Leonardo, il più giovane del gruppo, avvia questa specifica parte del dialogo, che richiama esplicitamente il Simposio platonico. Si susseguono quindi i discorsi di Leonardo, Ernesto ed Edoardo, fino all'arrivo di Giuseppe, che interrompe questa "tenzone" intorno al Natale, affermando: "[...] io non sono venuto a tenere discorsi, ma per godere con voi; e voi, per esprimermi lealmente, mi sembrate strani e quasi folli, in quanto fate qualcosa di simile". Con le parole di Giuseppe, che vede in Leonardo "[...] l'uomo pensante,

\footnotetext{
${ }^{11}$ Ibid., p. 111.

${ }^{12}$ Ibid., p. 116.

${ }^{13}$ Ibid., p. 117.
} 
riflettente, dialettico, iperrazionale" - in breve, cioè il "principio cattivo" - la festa riprende richiamando l'affinità del Natale con la fanciullezza.

Questa parte è certamente la più difficile da interpretare, non solo a livello contestuale, in quanto i personaggi non sono univocamente definiti, ma anche a un livello interpretativo superiore, di cui parleremo in seguito.

Tra i molti tentativi di dare un volto ai protagonisti del dialogo le ipotesi più verosimili hanno identificato in Ernestina Louise Reichardt, figlia del compositore Johann Friedrich Reichardt. Questa ipotesi sarebbe confermata anche dal fatto che Reichardt aveva dato un contributo alla Kirchenmusik, al quale Schleiermacher allude parlando di Sofia. Decisivo è però, per questa attribuzione d'identità, anche un altro elemento: Reichardt e la figlia furono i primi a mettere in musica la poesia romantica. È questo il primo indizio evidente che, al di là dell'immediato riferimento biografico, la scelta dei personaggi nasconde uno specifico interesse filosofico: come già annuncia la figura di Sofia, il legame tra musica e poesia romantica ci conduce nuovamente al tema del rapporto tra l'espressione del sentimento religioso e l'arte.

D'altro canto la figura di Ernestina richiama anche, come lo stesso Schleirmacher afferma, l'Ernestina delle Lettera sulla Lucinde, opera che il teologo aveva composto in difesa dell'amico Friedrich Schlegel, autore della Lucinde, il romanzo scandalo di fine Settecento ${ }^{14}$. Al di là dell'identificazione specifica della figura di Ernestina, il quadro generale rimane immutato: ciò che i racconti prima e i discorsi poi ci propongono è una ampia presentazione dei vari elementi che concorrono a formare la visione romantica del sentimento, dell'arte e della religione.

Leonardo, Ernesto ed Edoardo non rendono facile una immediata identificazione con personaggi dell'epoca. Le tre figure maschili conducono ad un livello autointerpretativo che fa di questa opera di Schleiermacher un momento assai particolare della sua produzione. Dal punto di vista del discorso strettamente teologico, sottolinea Karl Barth, Schleiermacher sarebbe Edoardo ${ }^{15}$. Tuttavia, in ognuno dei discorsi degli uomini, al di là

${ }^{14}$ Cf. SCHLEIERMACHER, Friedrich. Vertraute Briefe über Friedrich Schlegels Lucinde, LübeckLeipzig: Bohn, 1800; e SCHLEGEL, Friedrich. Lucinde. Bekenntnisse eines Ungeschickten. Berlin: Fröhlich, 1799 (tr.it. Lucinda, Pordenone: Studi tesi, 992). Pagine molto significative sulla difesa di Schlegel da parte di Schleiermacher in DILTHEY, Wilhelm. La vita di Schleiermacher, I. A cura di Francesca D’Alberto. Napoli: Liguori, 2008.

${ }^{15}$ Cf. BARTH, Karl. Schleiermachers "Weihnachtsfeier". In Id., Gesammelte Vorträge II: Die Theologie und die Kirche. Kaiser, München 1928. 
dei temi teologici, che intrecciano questioni fondamentali della teologia schleiermacheriana, si ritrovano elementi filosofici che rendono complessa una identificazione univoca dei protagonisti.

Dal punto di vista filosofico, a mio avviso, è Leonardo, der Ungläubige, a svolgere il ruolo più importante e decisivo per comprendere il senso dell'opera. Egli incarna, per così dire, l'elemento critico che viene costantemente attaccato, ma attorno a cui si struttura il cuore del dialogo. Già nel suo primo polemico intervento, Leonardo sottolinea che egli onora e ama "[...] quel che c'è di bello nella religiosità; essa però deve essere e restare qualcosa di intimo. Se essa vuole uscire all'esterno e creare particolari condizioni di vita, non fa che dar vita alla cosa più odiosa, all'orgoglio spirituale, che in fondo non è altro che la superstizione più bizzarra ed esaltata" ${ }^{16}$.

La polemica di Leonardo contro l'esibizione della religiosità non vuole colpire solo alcuni eccessi presenti nel pietismo, contro i quali si scaglia immediatamente non appena si rende conto dell'inclinazione pietistica dominante in Sofia, ma soprattutto colpire le tendenze cattolicizzanti presenti in esso. Corruzione e superstizione, diffuse in tutto il ceto ecclesiastico, sottolinea Leonardo, tra i cattolici conducono addirittura a una "ubriacatura" che coinvolge anche i laici, "[...] in virtù delle loro opere pie, le quali hanno appunto soltanto un valore esteriore" 17 .

L'attacco di Leonardo all'elemento irrazionale e superstizioso della religione non significa tout court, come sottolinea Edoardo, una adesione all'illuminismo ateo. Tutt'altro: Leonardo si preoccupa di salvare la religione dalla sua riduzione ad arte. Le sue argomentazioni non possono non ricordare le Reden, nelle quali Schleiermacher mostrava la necessità di rendere la religione indipendente dalla morale. Anche per Leonardo, che, mi pare, riveli delle argomentazioni molto vicine a quelle dell'autore, la religione non deve appoggiarsi a nessun'altra forma esteriore: per questo egli può affermare “[...] come cristiano sono molto poco artista e come artista sono molto poco cristiano"18. E ancor più esplicitamente egli ammette di non amare "[...] la chiesa rigida che Schlegel ci ha descritto nelle sue rigide stanze, e neppure le povere raggelate mendicanti arti, che sono liete di trovare un impiego" ${ }^{19}$.

\footnotetext{
${ }^{16}$ La festa di Natale, p. 82.

${ }^{17}$ Ibid., p. 82.

${ }^{18}$ Ibid., p. 86.

${ }^{19}$ Ibidem.
} 
Leonardo fa proprie alcune posizioni di Schleiermacher e polemizza direttamente con il maggior rappresentante del romanticismo e più intimo amico di Schleiermacher. In questo "attacco" diretto agli esiti della teoria romantica del legame tra religione e arte va riconosciuto il motivo più profondo che anima questo dialogo e che è "nascosto" dalle molte argomentazioni, che si sovrappongono a questo nucleo centrale.

Le affermazioni di Leonardo ci conducono dunque nel cuore dell'argomentazione: l'arte e la religione, nell'ottica qui proposta, vanno separate, perché la loro unione, sostenuta e incoraggiata dai romantici, segna l'inizio della fine della religione. La religione artistica, questo il cuore del discorso di Leonardo, conduce infine al cattolicesimo. Per quanto tutti gli altri protagonisti critichino Leonardo e intervengano a limare la sua posizione drastica, le sue idee non vengono mai radicalmente confutate dagli altri.

Illuminismo e pietismo sono i punti di riferimento del discorso di Leonardo. Se si entra nello specifico della sua argomentazione, si può notare come, alla rivalutazione dell'istanza comunitaria e sentimentale del pietismo, si accompagni una accesa difesa della parola come inevitabile mezzo di chiarificazione. Questi due elementi, illuminismo e senso della comunità, tracciano i contorni di una figura piuttosto complessa, che a maggior ragione può essere identificata con Schleiermacher. Egli infatti, pur avendo rotto con la comunità pietistica di Herrnhut, amava definirsi un "herrnhutiano di ordine superiore", evidenziando in questo modo la continuità tra la sua fase giovanile e l'influsso illuministico prima e romantico poi. In tal senso, la musica è ai suoi occhi certamente l'espressione più adatta per la religione, tuttavia essa è accompagnata da una fase preparatoria, in cui la narrazione (Erzaehlung), la parola è fondamentale.

Gli altri due discorsi vengono tenuti da Ernesto e da Edoardo. Il primo si sofferma sul fatto che i doni sono auspicio di progressi e begli avvenimenti, $\mathrm{e}$ «[...] la festa è l'annuncio di una nuova vita per il mondo». La festa annuncia quindi un nuovo stadio della vita e produce un preciso stato d'animo (Gemütszustand), non solo sereno e felice, ma anche commosso ed esaltato. Rispetto ad ogni altra festa, "[...] la peculiarità della gioia natalizia consiste in questa illimitata universalità", afferma Ernesto, e "ciò che è universale non può mai venire inventato arbitrariamente" ${ }^{20}$. L'intimità del sentimento religioso

${ }^{20}$ Ibid., p. 129. 
sostenuta da Leonardo viene dunque riconosciuta anche da Ernesto, ma accolta entro una visione universale condivisa dalla comunità.

Edoardo, come già accennato, presenta una pura argomentazione teologica: egli riprende la questione dell'elemento storico di Cristo, sottolineato nel discorso di Leonardo, per affermare invece il predominio dell'elemento mistico. Non il bambino è oggetto della festa, ma il Verbo divenuto carne: "[...] il Verbo è il pensiero, il conoscere; e il suo divenire carne è il manifestarsi di questa realtà originaria e divina in quella figura. Noi, quindi, non celebriamo nient'altro che noi stessi, quali siamo nella nostra totalità, o la natura umana" ${ }^{21}$.

I tre discorsi, in realtà, presentano punti di vista differenti in parte condivisi dall'autore stesso, le cui posizioni non sono né puramente speculative, né assolutamente critiche della espressione artistica della religione come quella di Leonardo. Dal punto di vista teologico, si può affermare che i tre discorsi siano complementari e rappresentino ognuno un aspetto specifico della riflessione teologica schleiermacheriana. È dal punto di vista filosofico, invece, che questa sostanziale armonia tra discorsi, nella quale non c'è un vincitore, viene meno e lascia spazio ad una presa di posizione più netta da parte dell'autore.

\section{LA NOVITÀ DEL DIALOGO: IL LIVELLO AUTOINTERPRETATIVO}

Una volta identificati i protagonisti e ricostruito il contesto storicobiografico dell'opera, il dialogo non ha ancora rivelato il suo senso. Certamente è possibile pensare che Schleiermacher volesse semplicemente rappresentare, attraverso i diversi protagonisti, le diverse posizioni e opinioni in materia religiosa e teologica. Sotto questo punto di vista, come già faceva notare Schelling, il modello di scrittura qui realizzato si avvicinerebbe più ai Topici di Aristotele che al Simposio di Platone. Proprio il dubbio relativo a quale modello di scrittura sia stato messo in opera da Schleiermacher spinge necessariamente a un livello interpretativo superiore.

Se, infatti, il modello fosse quello dei Topici, avremmo a che fare con una semplice esposizioni di posizioni date, tra le quali potremmo in teoria trovare anche quella di Schleiermacher, che, come si accennava, troverebbe forse nel contraddittorio Leonardo la sua identificazione più opportuna.

${ }^{21}$ Ibid., p. 335. 
Tuttavia il modello platonico esplicitamente richiamato all'interno del testo fa pensare che, oltre questa possibilità più lineare, si nasconde dietro alle singole posizioni una volontà di avvicinamento alla verità, in coerente stile platonico, che carica il livello biografico di un valore differente. La lettura biografica conduce a un livello interpretativo superiore, che richiama non solo la vita di Schleiermacher, ma anche la sua riflessione etica, che all'esperienza vitale era strettamente legata.

È noto che, accanto alle opere strettamente critiche o sistematiche, Schleiermacher aveva tentato ripetutamente di dare forma letteraria alla materia filosofica e teologica, come già testimoniano le Reden e i Monologen. Accanto a queste, che sono le sue opere più significative, egli aveva più volte cercato di scrivere novelle, come testimoniano i progetti ritrovati tra le sue carte. Se il legame tra forma e contenuto, tra scrittura e filosofia, era una costante della riflessione romantica, La festa di Natale cerca di creare una forma dialogica capace di riconoscere il valore del singolo protagonista e di darne una rappresentazione ben individualizzata (superando in tal senso la struttura dei dialoghi platonici, in cui solitamente è la figura di Socrate a spiccare e ad avere un'individualità ben definitiva).

Tre elementi vanno dunque ben tenuti in considerazione per cogliere al meglio questo ulteriore livello interpretativo: a) in primo luogo Schleiermacher intende dare spazio alle singole individualità, e perciò il riferimento ai personaggi dell'epoca è fondamentale. Il legame concreto con la realtà del tempo lo aiutava a conferire tratti realistici e ben individualizzati ai singoli protagonisti. b) In secondo luogo va tenuto presente che questo dialogo è concepito come un dono, che doveva essere scoperto da coloro che erano intimi. Questo fattore è fondamentale per comprendere l'importanza dell'opera. Il fatto che l'autore non venisse riconosciuto dalla cerchia di amici intimi alla quale si rivolgeva, o il fatto che non venisse apprezzato, significava la fine della Geselligkeit. Questo secondo elemento è particolarmente importante, perché conduce al terzo livello di lettura. c) Quando Schleiermacher scrive questo dialogo, ha già dietro di sé una intensa riflessione etica, iniziata negli anni giovanili. I temi affrontati da Schleiermacher rimandavano alla riflessione etica, più tardi raccolta nell' Entwurf eines Systems der Sittenlehre.

Arte, religione e Geselligkeit costituivano, secondo Schleiermacher, le forme dell' attività produttiva o dell'attività simbolica della ragione. La musica, come già visto, era la forma d'arte particolarmente legata alla religione. La sua caratteristica, ben evidenziata da Giuseppe, è l'assenza di parole. Confrontando 
la parte avuta dalle donne e quella sostenuta dagli uomini nel corso della festa, egli afferma: "[...] le povere donne si sono dovute sorbire tutto questo. Basterebbe pensare quali bei canti esse vi avrebbero potuto cantare, canti che sarebbero stati di gran lunga più intimamente pervasi di tutta la pietà dei vostri discorsi [...] il che vi avrebbe soddisfatto e ricreato meglio di quanto non siano riusciti a fare nei loro confronti questi discorsi solenni". E per annunciare il proprio punto di vista, Giuseppe sottolinea: "Oggi, per parte mia, non sono nemmeno in grado di recare aiuto con simili discorsi. Tutte le forme sono per me troppo rigide, e ogni discorso troppo noioso e freddo. L'argomento ineffabile richiede o produce anche in me una gioia silenziosa, e la mia gioia può soltanto ridere e giubilare alla maniera di un bambino"22.

Nelle parole di Giuseppe è nascosta una posizione decisiva del pensiero di Schleiermacher: il sacro non può trovare rappresentazione plastica o discorsiva, ma si trasmuta in elemento musicale. Giuseppe è stato identificato con August Gottlieb Spangenberg, vescovo herrnhutiano. Anche in questo caso, al di là dell'elemento biografico, non si può negare che in questa figura Schleiermacher rappresenti anche se stesso. La sua posizione potrebbe essere definita come la - apparentemente - contradditoria unione di Leonardo e Giuseppe, dell'illuminista e dell'herrnhutiano.

Nella ricomposizione di queste due figure avviene una svolta notevole nella posizione schleiermacheriana. Attraverso la figura di Giuseppe, Schleiermacher nega proprio i "discorsi": alla parola si sostituisce il canto e alle Reden, dove ancora arte e religione erano legate, subentra un nuovo progetto. La novità che stavamo cercando, come i protagonisti del dialogo cercano i doni di Natale velati da ciò che è apparentemente familiare, è la nuova fase di Schleiermacher, che abbandona il progetto artistico della giovinezza e inizia la sua fase sistematica.

Questa posizione, che creerà non pochi problemi all'interno dell'etica schleiermacheriana, in quanto rende complesso e in parte incoerente il rapporto tra arte e religione, come Dilthey ha messo in luce nel suo commento all'etica, portava a una rottura con gran parte delle idee del circolo romantico $^{23}$. In tal senso, il fatto che Schleiermacher chiami a chiudere il

22 Ibid., p. 140.

${ }^{23}$ Cf. DILTHEY, Wilhelm. Leben Schleiermachers. Zweiter Teil. Schleiermachers System als Philosophie und Theologie. Hrsg. von Martin Redeker. Berlin: De Gruyter, 1966. Su questo tema mi permetto di rimandare a Francesca D'Alberto, Ermeneutica e sistema. Dilthey lettore dell'etica di Schleiermacher. Padova: Cleup, 2011. 
dialogo un rappresentante della comunità herrnutiana significa una rottura e un distacco dalla cerchia romantica. Se, infatti, Schleiermacher, in gioventù, si era avvicinato al romanticismo, e in particolare a Friedrich Schlegel, uscendo dalla cerchia del pietismo herrnhutiano, con questo dialogo della maturità ritorna in parte sui suoi passi. L'eccessivo peso dato dai romantici all'elemento artistico rispetto a quello religioso viene in questo modo criticato con il richiamo alla religiosità musicale del pietismo.

Non è un caso che i romantici non apprezzarono questo dialogo, forse perché ben coglievano la critica in esso implicita alla loro concezione. La maggior parte di coloro ai quali il dialogo era rivolto non ne fecero menzione, altri ne diedero commenti negativi: come Achim von Arnim che definì la dialettica presente nel dialogo "un macinacaffe" e Clemens Brentano, che definì l'opera "uno stampo per cialde"24. Al di là dei giudizi negativi o del silenzio che calò sull'opera da parte di coloro che ne erano direttamente implicati, questa incomprensione significava, dal punto di vista di Schleiermacher, la rottura della Geselligkeit, i cui tratti egli andava definendo negli scritti di etica: significava quindi rottura di un legame intimo, basato sulla condivisione spirituale.

In tal senso si può ben intendere questo dialogo come il momento di frattura del legame con il circolo romantico e come opera di passaggio alla fase sistematica della riflessione schleiermacheriana, in cui i diversi ambiti della vita spirituale vengono identificati e analizzati come prodotti della natura umana nelle sue differenti attività. Come, in gioventù, Schleiermacher aveva distinto religione e morale, per poi chiarire la distinzione tra religione e metafisica (come avviene nella Dialettica), così ora, a partire da questo dialogo, distingue la religione dall'arte e avvia in modo coerente la sua riflessione etica sulle forme dell'agire umano, da quelle artistiche a quelle religiose.

${ }^{24}$ Cf. il saggio introduttivo di Moretto, p. 16. 
D'ALBERTO, Francesca. Autobiography and philosophical dialogue: the case of Schleiermacher's “Weihnachtsfeier. Ein Gespräch”. Trans/Form/Ação, Marília, v. 37, p. 7592, 2014. Edição Especial.

\begin{abstract}
Schleiermacher's Die Weihnachtsfeier. Ein Gespräch is one of the most interesting examples from the Romantic period of how literary form, vital and autobiographical context, and philosophical theses, constitute a unitary complex which is often difficult to interpret. In the Schleiermacherian dialogue on Christmas Eve, two levels of historiographical reading can be distinguished: a first level deals mainly with the context, and enables the identification of biographical references and the actual environment alluded to by the author; a second level, closely related to the first but not necessarily reducible to it, has a "self-interpretative" value. On this second level of reading, Schleiermacher's short work proves to be more than just a casual text for Christmas 1806. It simultaneously hides and reveals a willingness to break with the Romantic circle to whom the dialogue is dedicated, and marks the beginning of a new and autonomous kind of reflection for the author.
\end{abstract}

KEYWORDS: Friedrich Schleiermacher. Music. Art. Religion. Romanticism.

\title{
BIBILIOGRAFIA
}

BARTH, Karl. Die Theologie Schleiermachers 1923/24. A cura di D. Ritschl. Zürich: Theologischer Verlag, 1978.

. Schleiermachers "Weihnachtsfeier". In: Gesammelte Vorträge. München: Kaiser, 1928. v. 2: Die Theologie und die Kirche.

D'ALBERTO, Francesca. Ermeneutica e sistema: Dilthey lettore dell'etica di Schleiemacher. Padova: Cleup, 2011.

DILTHEY, Wilhelm. Leben Schleiermachers. In: . Gesammelte Schriften. Hrsg. von Martin Redeker. Berlin: Walter de Gruyter, 1970. v. 13. Tr. it. a cura di Francesca D’Alberto. Napoli: Liguori, 2008-2010.

Leben Schleiermachers: Zweiter Teil: Schleiermachers System als Philosophie und Theologie. In:__ Gesammelte Schriften. Hrsg. von Martin Redeker. Berlin: Walter de Gruyter, 1966. v. 14.

PATSCH, Hermann. Alle Menschen sind Künstler: Friedrich Schleiermachers poetische Versuche. Berlin: Walter de Gruyter, 1986.

. Metamorphosen des Erdgeistes. Neues Atheneum, v. 1, p. 248-279, 1989.

RICHARDSON, Ruth (Ed.). Schleiermacher in context. Lewinston: Edward Mellen Press, 1991.

SCHLEGEL, Friedrich. Lucinde. Bekenntnisse eines Ungeschickten. Fröhlich: Berlin, 1799.

Tr.it.: Lucinda. Pordenone: Studio Tesi, 1992. 
SCHLEIERMACHER, Friedrich, Monologen: Ein Neujahrsgabe. Berlin: Spener, 1800. Tr.it.: Monologhi. A cura di Ferruccio Andolfi. Reggio Emilia: Diabasis, 2011.

Meiner, 1981. Brouillon zur Ethik (1805-1806). Hrsg. von Hans-Joachim Birkner. Hamburg: . Über di Religion: Reden an die Gebildeten unter ihren Verächtern. Berlin: Unger, 1799. Tr. It.: Sulla religione: discorsi a quegli intellettuali che la disprezzano. A cura di Salvatore Spera. Brescia: Queriniana, 2005². - Vertraute Briefe über Lucinde Friedrich Schlegels. Berlin: Maurer, 1800.

SCHOLTZ, Gunter. Schleiermacher e la musica. In: MORETTO, G.; VENTURELLI, D. (A cura di). Filosofia religione nichilismo. Napoli: Morano, 1988. . Schleiermachers Musikphilosophie. Göttingen: Vandenhoeck\&Ruprecht, 1981.

SELGE, Kurt-Victor (hrsg.). Internationaler Schleiermacher-Kongress Berlin 1984. Berlin: Walter de Gruyter, 1985. 
D'ALBERTO, F. 